MS26-03

\section{Transitions toward complex electronic states and superperiodic structures in $\mathbf{P}_{4} \mathbf{W}_{16} \mathbf{O}_{56}$}

Elen Duverger-Nédellec ${ }^{1}$, Alain Pautrat ${ }^{2}$, Olivier Pérez ${ }^{2}$

1. XRD department, Charles University, Prague, Czech Republic

2. laboratoire CRISMAT, UMR 6508 CNRS, Caen, France

email: elenduvergernedellec@gmail.com

The MonoPhosphate Tungsten Bronzes (MPTB) family, $\left(\mathrm{PO}_{2}\right)_{4}\left(\mathrm{WO}_{3}\right)_{2 \mathrm{~m}}$, can be described by a regular intergrowth of $\left(\mathrm{PO}_{4}\right)$ tetrahedral layers and of slabs constituted by corner-sharing- $\left(\mathrm{WO}_{6}\right)$ octahedra, with a thickness depending on the $\mathrm{m}$ parameter. These low-dimensional oxides are known to exhibit successive transitions toward Charge Density Wave (CDW) states. These transitions are associated to lattice distortions leading to the appearance of incommensurate or commensurate structural modulations [1].In this family, the electronic anisotropy and the density of carriers of the system can be tuned by modifying the thickness of the $\mathrm{WO}_{3}$ slabs, i.e.changing $\mathrm{m}$. MPTB family is thus a relevant system to analyse the effect of the dimensionality on the CDW electronic instabilities. Temperature-dependant X-Ray Diffraction (XRD) [1] and transport measurements reported in the literature, for different terms of the family, reveal a significant change of behaviour between the terms with a low and high value of $m, m<7$ and $m>7$ respectively. Classical CDW transitions are reported for the low terms, characterized by a smooth resistivity jump and by the formation of clusters of tungsten in the centre of the $\mathrm{WO}_{3}$ slabs [2]. For the high terms [3], a structural transition is observed in XRD but the electronic transport studies do not show the usual signature attributed to a CDW. Moreover, the only structural study performed on a high term in the modulated state $(m=10)$ [3] evidences anti-ferroelectric-type (AFE) atomic displacements for the tungsten atoms without reporting of the formation of clusters of tungsten.

We will present both the transport properties and the analysis of the structural modulations for the $m=8$ term. Three first-order transitions associated with large thermic hysteresis were identified. The analysis of the structural modulations characterizing the different states, via the use of the super-space formalism, reveals the existence of AFE-type displacements and the formation of clusters for the tungsten atoms. These signatures can be assigned to the coexistence of AFE and CDW properties in the material. These two properties are a priori incompatible, but an extensive study of the transport properties versus temperature supports this hypothesis. This result enlightens the very interesting position of $\mathrm{P}_{4} \mathrm{~W}_{16} \mathrm{O}_{56}(m=8)$ in the border area between the low and the high $\mathrm{m}$ values in the MPTB family to discuss the competition regime between CDW and ferroelectric instabilities.

References:

[1] Ottolenghi, A. \& Pouget, J. P. (1996). J. Phys. I France, 6, 1059-1083

[2] Lüdecke, J. et al. (2000). Europhys.Lett., 49, 357-361

[3] Roussel, P. et al. (2000). Phys. Rev. B, 62, 176

Keywords: Modulations; Charge-density-wave; Anti-

ferroelectricity
MS26-04

\section{Protein crystal with ninefold structure modulation and 36 protein molecules in the asymmetric unit}

Joanna Śmietańska ${ }^{1}$, Joanna Śliwiak ${ }^{2}$, Mariusz Jaskólski², Mirosław Gilski $^{2}, Z^{2}$ bigniew Dauter ${ }^{3}$, Radosław Strzałka ${ }^{1}$, Janusz Wolny ${ }^{1}$

1. Faculty of Physics and Applied Computer Science, AGH University of Science and Technology, Krakow, Poland

2. Center for Biocrystallographic Research, Institute of Bioorganic Chemistry, Polish Academy of Sciences/Department of Crystallography, Faculty of Chemistry, A. Mickiewicz University, Poznan, Polande

3. Synchrotron Radiation Research Section, MCL, National Cancer Institute, Argonne National Laboratory, Argonne, United States of America

mail: j.smietanska@autograf.pl

Crystal structure modulation is manifested by the disappearance of short-range translational order with long-range order restored by an atomic modulation function (AMF). In the case of commensurate modulation, when the distortion remains in an integer-multiple relationship with the main lattice, the structure can be described in an expanded supercell or using the superspace concept. On the other hand, incommensurate modulation can be rigorously described only in a higher-dimensional superspace. A physical manifestation of a crystal with structure modulation is the presence of weak satellite reflections around the main Bragg reflections. Although this phenomenon is quite widespread and well-understood in small-molecule crystallography, only a limited number of commensurately modulated crystal structures have been successfully analyzed using the superspace approach [1]. In macromolecular crystallography this phenomenon is practically unheard of. The first successful structure solution and refinement of a modulated protein crystal structure was presented some time ago by us for the Hypericum perforatum PR-10 protein Hyp-1, which in that experiment was crystallized (as a tetartohedral twin) in complex with the fluorescent dye 8-anilino-1-naphthalenesulfonate (ANS) with apparent seven-fold nodulation (and 28 unique protein molecules in the expanded supercell) along the $\mathrm{c}$ direction of the $\mathrm{C} 2$ space group [2]. In the present project, the same protein was crystallized using different conditions to produce Hyp-1/ANS crystals with nine-fold c-parameter expansion of the same basic C2 unit cell and with 36 protein molecules in the asymmetric part of the expanded supercell. The structure analysis is complicated not only by the length of the c parameter $(\sim 400 \AA)$ but also by the tetartohedral twinning of the crystals. Using synchrotron X-ray diffraction data extending to $2.3 \AA$ resolution, the structure was solved by molecular replacement and is being refined, using maximum-likelihood targets, in the expanded supercell under the assumption of commensurate modulation.

Keywords: protein, modulation, phytohormone 